\title{
A Novel Approach to Blind I/Q Mismatch \& Carrier Offset Compensation
}

\author{
Sunita Panda ${ }^{1}$, Sasmita Kumari Padhy ${ }^{2}$, Siba P. Panigrahi ${ }^{3}$ \\ ${ }^{1}$ ECE, KIT, Berhampur, India; ${ }^{2}$ ITER, SOA University, Bhubaneswar, India; ${ }^{3}$ Electrical Engineering, KIST, Bhubaneswar, India. \\ Email: siba_panigrahy15@rediffmail.com
}

Received December $10^{\text {th }}, 2010$; revised January $11^{\text {th }}, 2011$; accepted February $18^{\text {th }}, 2011$

\begin{abstract}
This paper proposes a novel approach for $I / Q$ mismatch compensation. Simulation results proves the superior performance of the proposed technique.
\end{abstract}

Keywords: Channel Equalization, I/Q Mismatch Compensation

\section{Introduction}

In order to increase the receiver flexibility while also emphasizing the receiver integrability and other implementation related aspects, the design of radio receivers is no longer dominated by the traditional superheterodyne architecture. Instead, alternative receiver structures, like the direct conversion [1,2] and low-IF [1,3,4] architectures, are receiving more and more interest. The analog front-end of these types of receivers is partially based on complex or I/Q signal processing [5-7]. More specifically, the frequency translation from radio frequencies (RF) closer to base-band is carried out using $\mathrm{I} / \mathrm{Q}$ downconversion. Since, in theory, the I/Q downconversion corresponds to a pure frequency translation, the fundamental image signal problem is basically avoided during the downconversion. In thismanner, the requirements for RF image rejection filtering are greatly relaxed in practice [1-4].

The assumption of infinite image signal rejection during the downconversion is strictly valid only if amplitudes and phases of the analog front-end I and Q branches are perfectly matched [7]. In practice, this is not the case. Some mismatch or imbalance will always exist due to imperfections of practical analog electronics. Amplitude imbalances of $1 \%-5 \%$ and phase imbalances of 1-5 represent feasible design Figures [1-4]. This corresponds to approximately $25-40 \mathrm{~dB}$ image signal attenuation. These levels of image attenuation are clearly insufficient in low-IF-type receivers where the image band can carry a signal with much higher power level than the desired signal. Some digital signal processing (DSP)- based approaches to improve this image attenuation in IF receivers are presented, for example, in [7-10]. In direct-conversion receivers, the image signal is inherently a self-image (the desired signal itself at negative frequencies), and the analog front-end image attenuation might be sufficient with low-order modulations.

However, with higher-order modulations, such as 16or 64-QAM, the distortion due to self-image cannot be neglected and again some kind of compensation is needed [11-13]. This is also one of the topics of this paper. The idea in this paper is first to show that I/Q mismatch causes crosstalk between the I and Q components. Then this crosstalk or mixing of the I and Q is removed using blind signal separation (BSS) techniques [14-16]. Compared to the other available solutions in the literature $[12,13]$, the proposed concept is especially attractive since no known training signals are needed. Also the ability to follow possible time dependencies in the mismatch parameters (due to, e.g., temperature changes) is another highly desirable feature.

Another challenging practical problem in radio communications is carrier synchronization [6]. In practice, it is unlikely that the frequency and relative phase of the receiver local oscillators exactly match those of the received carrier. In case of linear modulations, a constant phase offset introduces a constant rotation to the received constellation, which needs to be compensated unless differential phase modulation is used. Even a bigger problem is caused by errors or offsets in frequency that basically results in time-varying rotation of the constellation. This is obviously unacceptable for most modulation types and needs to be efficiently compensated for. In this 
paper, the carrier offsets are shown to result in time- varying leakage between the I and Q, and carrier tracking is implemented with adaptive signal separation methods. Furthermore, when combined with the previous I/Q mismatch compensation, a single BSS stage can accomplish both tasks jointly in a blind manner. These kinds of approaches have not been considered in the literature so far.

In addition to the above front-end-related issues, the distortion due to transmission channel $[6,15]$ is inevitable in any radio receiver and needs to be addressed with care. As will be shown, a general bandpass channel can be viewed to cause frequency-selective crosstalk between the transmitted I and Q data. Based on this, convolutive mixture (or FIR-MIMO) separation techniques working on the observed I and Q signals are applied to implement channel equalization.

\section{Direct-Conversion Receiver}

\subsection{Background}

The fundamental tasks to be carried out in the front-end of any communications receiver include 1) amplification of the attenuated desired signal, 2) downconversion of the desired signal spectrum from around the RF carrier frequency closer to base-band, 3) attenuating the unwanted spectral components appearing in the antenna signal, and 4) synchronizing the receiver local oscillators for downconversion and sampling with the received signal. Key tasks of the baseband digital signal processing include channel estimation, equalization, and detection. In traditional receiver designs, these tasks are implemented more or less independently of each other, aiming at close-to-ideal operation in each signal processing stage. This has lead to the use of the superheterodyne receiver architecture $[1,4]$ in order to meet the tight RF specifications in terms of image band attenuation and nonlinear effects of the receiver front-end stages. In such receivers, the RF signal is first downconverted to a fixed intermediate frequency (IF) where the receiver selectivity is implemented using a fixed IF filter. The down-conversion is usually done with a simple real mixer, and the spectral components on the image signal band need to be attenuated sufficiently by the RF stages before the mixer. Due to the high number of discrete components and high power consumption, the superheterodyne architecture is, however, not the most appropriate choice for highly integrated implementations [1-4].

Furthermore, the use of fixed discrete components in the analog front-end limits the receiver flexibility. Thus, architectures with more simplified analog front-ends with less RF processing are generally needed. In addition, it has recently been demonstrated [7-9] that various non-idealities and distortion effects due to the simplification of the analog front-end can in general be compensated by advanced DSP techniques. This is also the central theme in this paper.

\subsection{Direct-Conversion Architecture}

A simple way to reduce the number of components in the receiver and alleviate the problem of receiver complexity is to avoid the use of intermediate frequency and quadrature down-convert the desired channel signal directly from RF to baseband. Complete elimination of the IF stage results in highly simplified structure, the so-called direct-conversion receiver, where most of the channel selectivity and amplification are implemented at baseband [1,2]. Firstly, since most of the signal processing tasks take place at low frequencies, the power consumption is minimized. On the other part, very low-noise operation is called for in all the remaining analog components since the amplification provided by the RF stage is only moderate. The direct-conversion receiver concept is depicted in Figure 1.

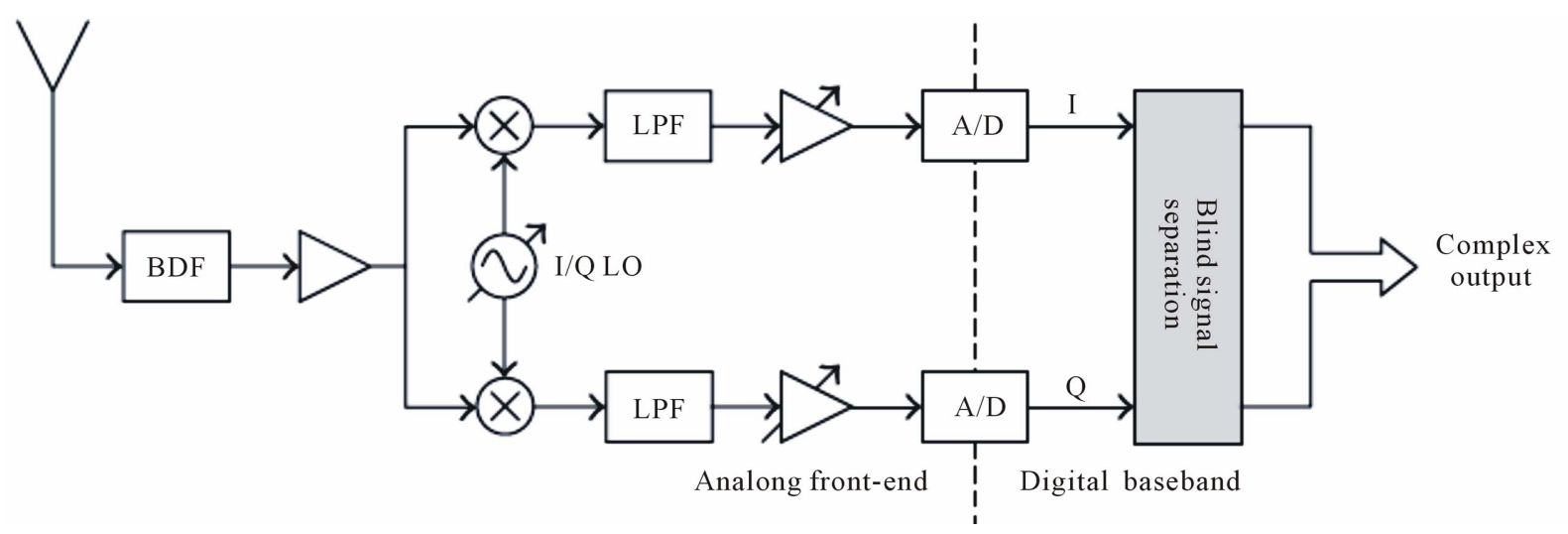

Figure 1. The direct-conversion receiver architecture. The leading principle in this paper is to show that various receiver signal processing tasks can be carried out blindly by forcing the observed $I$ and $Q$ signals as independent as possible using blind signal separation. 
In the direct-conversion principle, since the IF is effectively zero, the image signal is actually the desired signal itself (at negative center frequency). Ideally, with perfect analog processing, the image band is completely attenuated in general. However, practical analog implementations of the needed I/Q signal processing have mismatches in the amplitude and phase responses of the I and $\mathrm{Q}$ branches, leading to finite attenuation of the image band signal. In the direct-conversion case, the effect of imperfect (self-)image rejection is seen as a linear transformation of the original signal constellation [11-13]. As a result, the image attenuation requirements are not extremely tight, especially if low-order modulations are used. In effect, the $25-40 \mathrm{~dB}$ image attenuation of a practical analog front-end can be sufficient with low-orde modulations. With higher-order spectrally efficient modulation methods, however, the distortion due to selfimage can establish an error floor and needs to be compensated.

In practice, the use of zero IF introduces also some other problems. The major drawback in direct-conversion principle is the DC offset problem [1,2]. Due to zero IF, the local oscillator frequency is on the same frequency as the desired channel. Then, if the LO signal leaks into the mixer input port, it self-mixes down to baseband causing interfering signal components at zero frequency. These are called DC offsets and can be orders of magnitude larger than the desired channel signal. Besides the LO leakage, another contributor to the offset problems is $1 / f$ noise of the active front-end components. For a better receiver performance, some compensation of the DC offsets is needed. Another analog RF-related problem is that higher linearity is required because in a direct-conversion receiver, second-order inter-modulation products may fall in the signal band (in superheterodynes, the weaker third-order intermodulation products usually set the linearity requirements). These problems have limited the use of direct-conversion principle in practical systems earlier, but nowadays this approach is widely utilized in mobile terminals. However, the introduction of higherorder modulations in future wireless communication systems sets higher demands for the receiver performance, and the I/Q imbalance effects, for example, to be discussed in Section 4 are likely to pose big challenges.

\section{Blind Signal Separation}

Currently, more and more applications call for proper representation of multivariate data. An extension of principal component analysis (PCA) called independent component analysis (ICA) is one good example of such techniques [16]. The ICA and its signal processing application, blind signal separation $[14,16]$, is applied in this paper to communications receiver signal processing. The purpose of this section is to introduce the basic con- cepts and notations after which the actual applications to I/Q mismatch and carrier offset compensation as well as to channel equalization are discussed and analyzed in Sections 4 and 5.

Generally speaking, blind signal separation deals with the recovery of some interesting signals, called sources, based on observing their linear1 mixtures, and falls under the umbrella of multiple-input multiple-output (MIMO) signal processing [14-16]. The term blind is used here to emphasize the fact that no prior knowledge of the mixing process or the temporal structure of the underlying source signals is needed, but only the statistical properties are utilized. The leading principle in this context is the assumption of statistical independence of the original sources [14-16]. Thus in practice the recovery consists of forcing the separator output signals to be "as independent as possible," according to the selected independencemeasure. Commonly used approaches in this context are, for example, nonlinear decorrelation and minimization of mutual information. As is obvious, the relative order of the recovered sources or the individual amplitude/power levels cannot be blindly identified.

In addition to statistical independence, another key assumption is that only one of the sources (if any) can be Gaussian [14-17]. This is easy to understand since for Gaussian signals uncorrelatedness implies independence, making it impossible to distinguish any (remaining) orthogonal/orthonormal transformation. Luckily most communications signals are, indeed, non-Gaussian. In the following, $M$ and $N$ denote the number of observed and original source signals, respectively.

\subsection{Instantaneous MIMO Models}

Assuming the mixing process is instantaneous, the $m$ th observation, say $x_{m}(k)$ is of the form $x_{m}(k)=$ $a_{m, 1} s_{1}(k)+\cdots+a_{m, N} s_{N}(k)$, where, $x_{n}(k)$ denotes the $\mathrm{n}^{\text {th }}$ source signal and $a_{m, 1}$ represents the relative weight of the source $s_{n}(k)$ in the observation $x_{n}(k)$. Stacking the observed and source signal samples at time-instant $k$ into column vectors $s(k)=\left[s_{1}(k), \cdots s_{N}(k)\right]^{T}$ and $x(k)=\left[x_{1}(k), \cdots x_{N}(k)\right]^{T}$ respectively, the system model can be simply written as:

$$
x(k)=A s(k)
$$

where the $M \times N$ matrix A describes the mixing process with $[A]_{m, n}=a_{m, n}$. In general, the matrix $\mathbf{A}$ is assumed unknown but nonsingular (full rank). This is a natural assumption and is fundamental for the identifiability of the model; see [14,16-18] for more details. Generally speaking, the separator processes a sequence of the observation vectors and tries to "invert" the model in (1). In adaptive separation, the parameters of the separator are 
updated iteratively which also enables tracking the possible time-variant features of the mixturemodel. The separator output at time $k$ is commonly written as

$$
y(k)=W(k) x(k)=W(k) A s(k)=T(k) s(k)
$$

Where, $W(k)$ denotes the $N \times M$ separator matrix, $T(k)=W(k) A$ is the total equivalent system matrix $(N$ $\times N)$, and $y(k)=\left[y_{1}(k), \cdots y_{N}(k)\right]^{T}$. For successful separation, $\mathbf{T}(k)$ should converge to a "quasi-identity" (permutation and scaling) matrix with only one nonzero element on each of its row and column.

Various algorithms, with varying computational complexity and performance, to determine the separator coefficients exist in the literature; see $[14,16]$ for excellent reviews.

One exciting feature of the separation stage is the possibility for uniform performance. This being the case, the separation performance is independent of the underlying mixture coefficients (i.e., the matrix $\mathbf{A}$, as long as it is full rank) and depends only on the source statistics. One such algorithm with this desirable property is the so-called equivariant adaptive separation via independence (EASI), proposed originally in [19], whose performance depends only on the certain nonlinear moments of the source signals. The EASI algorithm consists formally of two subtasks; a whitening (second order decorrelation) part and a nonlinear decorrelation part where the selection of the used nonlinear function depends on the source statistics. The exact algorithm description can be found in [19]. This algorithm is used also in this paper in Section 4. Notice, however, that this is done only to illustrate the principal operation of the proposed receiver concepts; thus any other adaptive separation approach could be tested and used as well in practice.

\subsection{Convolutive MIMO Models}

A more general class of signal models is obtained if the assumption of instantaneous mixing is dropped. In other words, the mixing process can also containmemory and thus be frequency selective $[14,16,20]$. A direct extension of the model in (1) results in

$$
x(k)=\sum_{l} A_{l} s(k-l)
$$

Which represents a MIMO convolution of the sequences $\{\cdots, s(k-1), s(k) s(k+1, \cdots)\}$ and $\left\{\cdots, A_{-1}, A_{0}, A_{1}, \cdots\right\}$ with each $A_{l}$ being size $M \times N$. In other words, each observation $x_{n}(k)$ is a convolutive mixture of the original source signals. In this case, the identifiability condition related to the mixing process is generally formulated in terms of the corresponding system (MIMO) transfer function $A(z)=\sum_{l} A_{l} z^{-l}$; the recovery of the original source contributions is feasible if this system transfer function has full rank [14,16,20].

Practical recovery of the source signals consists of multichannel filtering of the observed vectors and can generally be written as:

$$
y(k)=\sum_{l} W_{l}(k) x(k-l)
$$

where, the $N \times M$ separator matrices $W_{l}(k)$ are adapted to minimize the predetermined dependence measure between the components of $\mathbf{y}(k)$. In terms of system transfer functions, the relation between the source signals and the separator output signals is of the form:

$$
T(z, k)=W(z, k) A(a)
$$

where, $\mathbf{W}(z, k)$ is the transfer function of the separator at time $k$. For successful source recovery, the total system $\mathbf{T}(z, k)$ should converge to a matrix with only one nonzero element in each row and column.

As in case of instantaneous mixtures, also here a wide variety of different algorithms for separator adaptation exists in the literature, and some of them can be claimed to have the uniform separation performance $[14,16,20]$. One example is the natural gradient-based approach described in [20]. This algorithm is applied also in this paper in Section 5 to the channel equalization problem in terms of the I and Q signals.

\section{Blind I/Q Mismatch \& Carrier Offset Compensation}

In this section, the I/Q mismatch problem due to analog front-end nonidealities as well as the carrier synchronation task are addressed in detail for direct-conversion receivers. The basic idea is to show that both of these practical problems can be viewed to create dependence between the observed I and Q signals. Then, a signal separation algorithm is applied to remove this dependence and thus to recover the original I and Q data..

\subsection{Signal Models and I/Q Separation-Based Compensation}

\section{I/Qmismatch:}

For analysis purposes, the received RF signal, say $r(t)$, is written as:

$$
\begin{aligned}
& r(t)=\operatorname{Re}\left[z(t) \exp \left(j \omega_{c} t\right)\right] \\
& \quad=z_{I}(t) \cos \left(\omega_{c} t\right)-z_{Q}(t) \sin \left(\omega_{c} t\right)
\end{aligned}
$$

where, $z(t)=z_{I}(t)+j z_{Q}(t)$ denotes the corresponding ideal baseband equivalent of the desired channel to be recovered by the receiver front-end. Now, to model the amplitude and phase mismatches of the analog front-end, the (complex) LO signal of the I/Q down- converter is written as: 


$$
\begin{aligned}
x_{L O}(t) & =\cos \left(\omega_{c} t\right)-j g \sin \left(\omega_{c} t+\varphi\right) \\
& =K_{1} \exp \left(-j \omega_{c} t\right)+K_{2} \exp \left(j \omega_{c} t\right) \\
K_{1}= & \frac{1+g \exp (-j \varphi)}{2}, K_{2}=\frac{1-g \exp (j \varphi)}{2}
\end{aligned}
$$

where, $g$ and $\varphi$ represent the relative amplitude and phase mismatches, respectively. The latter form of (7) indicates that two frequency translations take place due to mismatches. Indeed, the down-conversion of $r(t)$ combined with low-pass filtering results in:

$$
x(t)=K_{1} z(t)+K_{2} z *(t)
$$

and the corresponding self-image rejection ratio is: $10 \log _{10}\left(\left|K_{1}\right|^{2} /\left|K_{2}\right|^{2}\right)$.

To examine the mismatch effect from the I and Q signal point of view, the model in (9) can be written as $x(t)=x_{I}(t)+j x_{Q}(t)$, where,

$$
\begin{aligned}
& x_{I}(t)=z_{I}(t) \\
& x_{Q}(t)=g \cos (\varphi) z_{Q}(t)-g \sin (\varphi) z_{I}(t)
\end{aligned}
$$

In other words, I/Q mismatch tends to create dependence between the I and Q signals. Assuming that the original I and Q signals are statistically independent, which holds, for example, for square QAM type of constellations, these original I and Q components can be recovered blindly using a signal separation algorithm.

\section{Carrier offsets:}

To see the explicit effect of carrier offsets more formally, the $\mathrm{I} / \mathrm{Q}$ down-converter LO signal is now written as:

$$
\begin{aligned}
x_{L O}(t) & =\cos \left(\left(\omega_{c}+\Delta \omega\right) t+\theta\right)-j \sin \left(\left(\omega_{c}+\Delta \omega\right) t+\theta\right) \\
& =\exp \left(-j\left(\left(\omega_{c}+\Delta \omega\right) t+\theta\right)\right)
\end{aligned}
$$

where, $\Delta \omega$ and $\theta$ model the frequency and phase offsets, respectively, relative to the received signal in (6). Now, it is common to write the down-converted signal after lowpass filtering as:

$$
x(t)=z(t) \exp (-j(\Delta \omega t+\theta))
$$

Interestingly, when written in terms of the I and Q signals, the model in (12) can be expressed as:

$$
\begin{aligned}
& x_{I}(t)=\cos (\Delta \omega t+\theta) z_{I}(t)+j \sin (\Delta \omega t+\theta) z_{Q}(t) \\
& x_{Q}(t)=\cos (\Delta \omega t+\theta) z_{Q}(t)-j \sin (\Delta \omega t+\theta) z_{I}(t)
\end{aligned}
$$

Thus, from the I/Q point of view, the carrier offsets correspond to time-varying mixing of the I and Q signals, and an adaptive signal separation algorithm can be used to track and remove this effect.

A combined signal model incorporating both the $\mathrm{I} / \mathrm{Q}$ mismatch and the carrier offset effects is given next. Using analysis similar to those given above, it is relatively easy to show that observable signal after down-conversion and low-pass filtering appears as:

$$
\begin{aligned}
x(t) & =K_{1} z(t) \exp (-j(\Delta \omega t+\theta)) \\
& +K_{2} z^{*}(t) \exp (j(\Delta \omega t+\theta))
\end{aligned}
$$

Here it is naturally assumed that the frequency offset is smaller than the guard band between the adjacent frequency channels. Now, the complex signal in (14) corresponds to an $\mathrm{I} / \mathrm{Q}$ signal pair of the form:

$$
\begin{aligned}
x_{I}(t) & =\cos (\Delta \omega t+\theta) z_{I}(t)+\sin (\Delta \omega t+\theta) z_{Q}(t) \\
x_{Q}(t) & =g \cos (\Delta \omega t+\theta+\varphi) z_{Q}(t) \\
& -g \sin (\Delta \omega t+\theta+\varphi) z_{I}(t)
\end{aligned}
$$

\section{Joint compensation using blind I/Q signal separa- tion:}

As given in (15), the observable I and Q signals in the presence of I/Q mismatch and carrier offsets appear as instantaneous and time-varying mixtures of the true I and Q signals. Switching to discrete-time notations $x_{I}(k)=$ $x_{I}(k T s)$, and so forth, we introduce $2 \times 1$ source and observation vectors $s(k)=\left[z_{I}(k), z_{Q}(k)\right]^{T}$ and $x(k)=$ $\left[x_{I}(k), x_{Q}(k)\right]^{T}$ and write the model in $(15)$ as $\mathbf{x}(k)=$ $\overline{\mathbf{A}}(k) \mathbf{s}(k)$, where,

$$
A(k)=\left[\begin{array}{cc}
\cos (\Delta \omega k+\theta) & \sin (\Delta \omega k+\theta) \\
-g \sin (\Delta \omega k+\theta+\phi) & g \cos (\Delta \omega k+\theta+\phi)
\end{array}\right]
$$

Notice that the frequency offset $\Delta \omega$ refers here to the "normalized" frequency variable $\Delta \omega=2 \pi \Delta f / f S$. Now an adaptive signal separation algorithm, such as the EASI algorithm discussed in Section 3, can be used to blindly estimate the source signals $z I(k)$ and $z Q(k)$.

It is interesting to note that the identifiability of the model in (16) is independent of the carrier offset levels and also practically independent of the mismatch values. This can be seen more formally by examining the determinant of $\mathbf{A}(k)$ :

$$
\operatorname{det}(A(k))=g \cos \phi
$$

Thus, the system matrix $\mathbf{A}(k)$ is invertible given that $g \neq 0$ and $\phi \neq \pm \pi / 2$. The first requirement $(g \neq 0)$ simply states that the downconversion stage needs to produce two non-zero signals while the second one prevents the case where the two signals after down-conversion would be just scaled versions of each other. These are more than natural requirements for any I/Q front-end and are always fulfilled by any practical analog design. Thus this indicates that the proposed idea is robust in the face of dif- ferent imbalance and offset levels in terms of identifiability. 
There are some further practical issues related to the proposed compensation scheme. First of all, as discussed in Section 2, the direct-conversion architecture suffers from the well-known DC offset problem $[1,2,4]$ due to self-mixing of the LO signal leaking into the mixer RF port. Most signal separation algorithms, in turn, assume zero-mean data, so the DC offset needs to be compensated prior to the separation stage. Another practical aspect is related to the amount of frequency offsets tolerated. On one hand, the frequency offset should be smaller than the guard band between adjacent frequency channels. If not, the receiver is not anymore really zero IF but closer to low IF and the nearby channel signal (or at least part of it located on the true image band) appears as interference on top of the desired signal after downconversion. On the other hand, the frequency offset determines the dynamics of the system matrix $\mathbf{A}(k)$ in (16), which is indeed the dynamics that the adaptive separation algorithm needs to follow. In other words, this dynamics should be within the tracking capability of the applied adaptive algorithm. Commonly, this poses some limitations to the used step-sizes such that a relatively large stepsize is needed. The used step-size, in turn, is usually directly related to the separator steady-state performance and cannot, of course, exceed its own algorithm-specific stability limit $[14,16,19]$. Thus we can conclude that even though the frequency offset level is irrelevant from the identifiability point of view, the tracking capability of the practical algorithms as well as the role of the image signal limit the applicability of the proposed concept to mild frequency offset. In other words, coarse frequency synchronization should be implemented by other means prior to the separation stage. Notice also that due to the amplitude (sign) and ordering ambiguities mentioned in Section 3, it is possible that the recovered constellation still formally suffers from 1 ) a constant phase rotation of (integer multiple of) 90 and/or 2) complex conjugation. In practice, these issues can be easily resolved using a little side information in the actual data detection stage. It should be noted that any blind algorithm is subject to similar ambiguities in general.

As mentioned above, these ambiguities can be reduced in a later stage of the receiver, for example, by using a minimal number of known symbols (pilot or training symbols specified in the signaling frame structure) or by using differential coding/mapping between bits and symbols. In general, for modulations other than square QAM, the effects of possible dependence between the true I and $\mathrm{Q}$ should be explored individually.

\section{Simulations}

Here some example results are given to illustrate the efficiency of the proposed compensation idea. In the simu- lations, imbalance levels of $3 \%$ and 3 are used corresponding to an approximate of $30 \mathrm{~dB}$ image attenuation which should represent a typical practical case. Phase offset in the system is assumed to be 20 and the (remaining) frequency offset $0.0001 \times 2 \pi$. Given, for example, a $10 \mathrm{MHz}$ sampling frequency, this corresponds to $1 \mathrm{kHz}$ absolute frequency offset.

The data modulation is 16 QAM. The model also includes additive white Gaussian noise (AWGN) with the signal-to-noise ratio (SNR) ranging from $0 \mathrm{~dB}$ to $20 \mathrm{~dB}$. The EASI algorithm discussed in Section 3 is then used as an example algorithm in the separation stage with a step-size of 0.01 and a third-order (cubic) nonlinearity [19].

The time-varying mixture coefficients are illustrated in Figure 2(a), followed below by an example realization of the separator coefficients in Figure 2(b) with SNR of $20 \mathrm{~dB}$. Clearly, the separation algorithm is able to track the time- varying mixture coefficients successfully. The corresponding symbol rate output samples without and with compensation are depicted in Figure 3. As is evident, the signal without compensation is useless due to I/Q mismatch and carrier offsets. The compensator output signal, however, is a good estimate of the transmitted symbol constellation.

The most fundamental performance measure of any digital communication system is the bit or symbol error rate (BER/SER). This is assessed next for the proposed compensator as a function of additive noise SNR. The decisions are made symbol by symbol using the minimum distance detection principle. The obtained results are depicted in Figure 4 which also shows the SER with additive noise only for reference.

The corresponding SER without any compensation is close to one, independently of the SNR, and is not shown for simplicity. As is evident, the proposed compensator can efficiently estimate the transmitted signal, bringing the error rate close to the AWGN bound. Especially in the raw SER levels of $10^{-1}$ to $10^{-2}$, which is the crucial operating range of any practical system before errorcontrol decoding, the proposed receiver is really close (within $1 \mathrm{~dB}$ ) to the noise limit.

\section{Conclusions}

In this paper, blind I/Q signal separation-based approaches for receiver signal processing were proposed. More specifically, the I/Q mismatches and carrier offsets as well as the linear distortion due to general band pass channels were shown to create crosstalk between the transmitted I and Q signals.

Then compensation structures utilizing blind signal separation were used to compensate for these effects. Also some simulation results were given to illustrate the efficiency of the proposed techniques. Combining the 


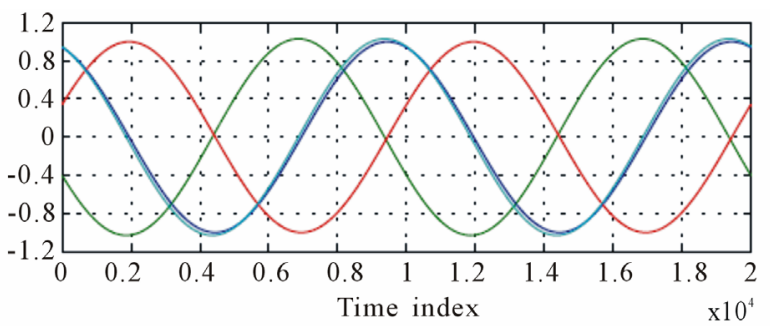

(a) Mixture coefficients.

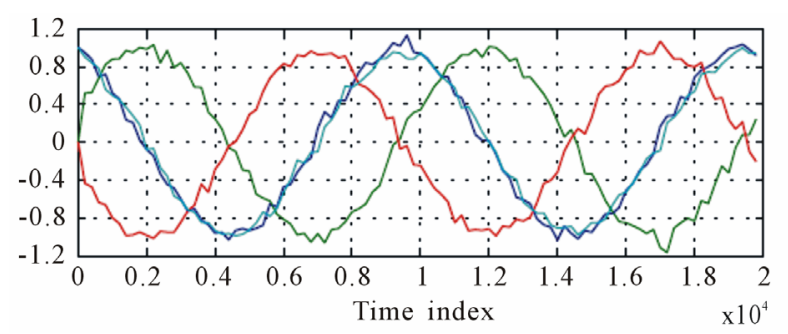

(b) Separator coefficients.

Figure 2. (a) An illustration of the dynamics of the system matrix. (b) One realization of the separator coefficients using the EASI algorithm (step-size 0.01). The I/Q mismatch values: $g=1.03$ and $\varphi=3$. The carrier offset levels: $\theta$ $=20$ and $\Delta \omega=2 \pi \times 0.0001$. Additive noise $\mathrm{SNR}=20 \mathrm{~dB}$.

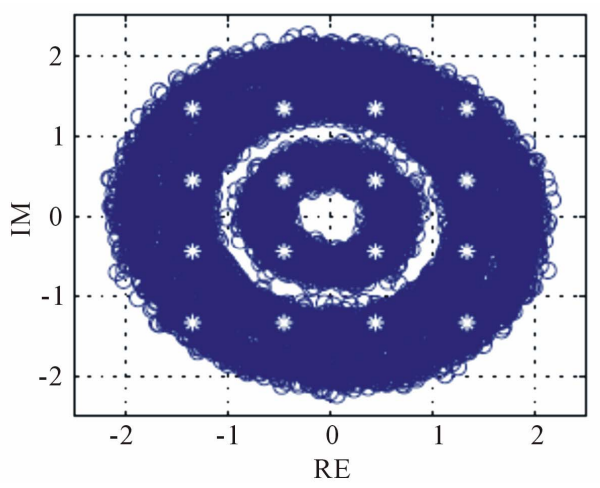

(a) Without compensation.

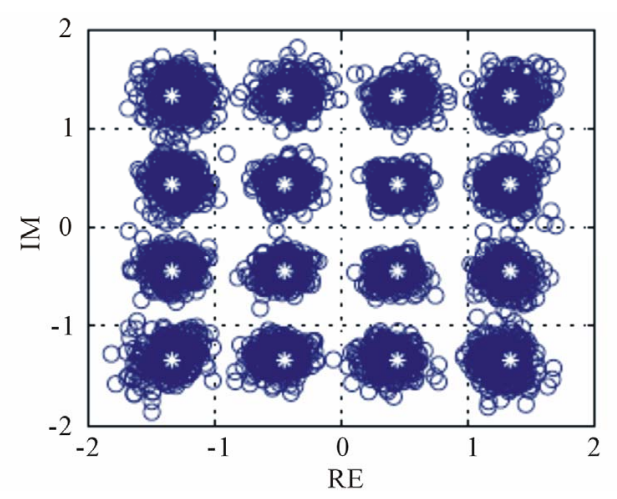

(b)With compensation.

Figure 3. Symbol rate output samples (16-QAM) (a) without and (b) with compensation. The I/Q mismatch values: $g=$ 1.03 and $\varphi=3$. The carrier offset levels: $\theta=20$ and $\Delta \omega=2 \pi$ $\times$ 0.0001. Additive noise $S N R=20 \mathrm{~dB}$.

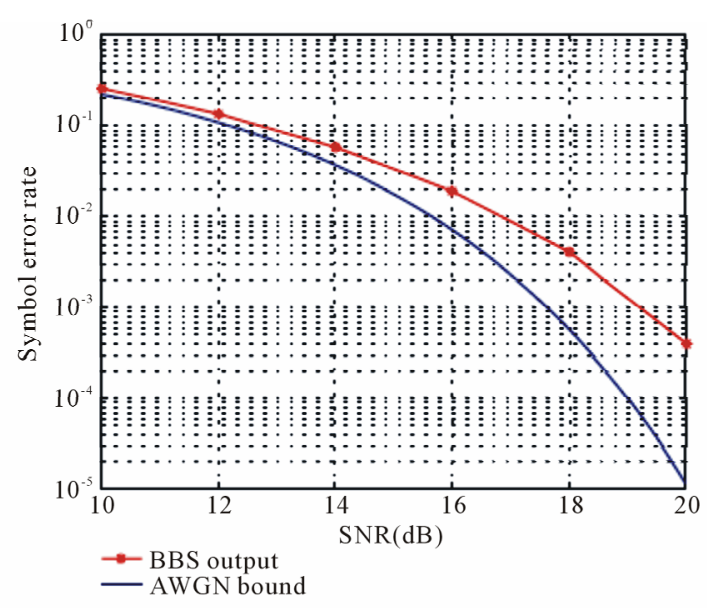

Figure 4. Symbol error rate of the EASI algorithm-based compensator for 16-QAM data. The I/Q mismatch values: $g$ $=1.03$ and $\varphi=3$. The carrier offset levels: $\theta=20$ and $\Delta \omega=$ $2 \pi \times \mathbf{0 . 0 0 0 1}$. Also shown for reference is the symbol error rate with additive noise only (AWGN bound).

presented I/Q mismatch and carrier offset compensation and the channel equalizer principles into a single (or a cascade of two) I/Q separator(s) results in a versatile receiver building block for future radio communication systems. Future work should be directed to further verification and prototyping of the proposed approaches using measured real-world receiver frontend signals.

Generally speaking, the idea behind this paper is to give new views for applying complex or I/Q signal processing efficiently in radio receiver design and to take full advantage of the rich signal structure inherent to complex-valued communications signals.

\section{REFERENCES}

[1] S. Mirabbasi and K. Martin, "Classical and Modern Receiver Architectures," IEEE Communication Magazine, Vol. 38, No. 11, 2000, pp. 132-139. doi:10.1109/35.883 502

[2] A. A. Abidi, "Direct-conversion Radio Transceivers for Digital Communications," IEEE Journal Solid-State Circuits, Vol. 30, No. 12, 1995, pp. 1399-1410. doi:10.1109 /4.482187

[3] J. Crols and M. S. J. Steyaert, "Low-IF Topologies for HighPerformance Analog Front Ends of Fully Integrated Receivers," IEEE Transaction Circuits System II, Vol. 45, No. 3, 1998, pp. 269-282. doi:10.1109/82.664233

[4] J. Crols and M. S. J. Steyaert, "CMOS Wireless Transceiver Design,” Kluwer Academic, Dordrecht, 1997.

[5] M. E. Frerking, "Digital Signal Processing in Communication Systems," Chapman \& Hall, New York, 1994.

[6] E. A. Lee and D. G. Messerschmitt, "Digital Communication," Kluwer Academic, Norwell, 1988.

[7] M. Valkama, "Advanced I/Q Signal Processing for Wi- 
deband Receivers: Models and Algorithms," Ph.D. Dissertation, Tampere University of Technology, Tampere, 2001.

[8] G. Fettweis, M. L“ohning, D. Petrovic, M. Windisch, P. Zillmann and E. Zimmermann, "Dirty RF," Proceeding on Wireless World Research Forum, Oslo, June 2004. pp. 133-148.

[9] M. Valkama, J. Pirskanen and M. Renfors, "Signal Processing Challenges for Applying Software Radio Principles in Future Wireless Terminals: an Overview," International Journal of Communication Systems, Vol. 15, 2002, pp. 741-769. doi:10.1002/dac.561

[10] J. P. F. Glas, "Digital I/Q Imbalance Compensation in a low-IF Receiver," IEEE Proceeding on Global Telecommunications Conference, Sydney, November 1998, Vol. 3, pp. 1461-1466.

[11] ] M. Valkama and M. Renfors, "Digital I/Q Imbalance Compensation in Direct-Conversion Receivers," Proceeding on Workshop on Software Radios, Karlsruhe, March 2002, pp. 51-55.

[12] I.-H. Sohn, E.-R. Jeong and Y. H. Lee, "Data-aided Approach to I/Q Mismatch and DC-offset Compensation in Communication Receivers," IEEE Communication Letters, Vol. 6, No. 12, 2002, pp. 547-549. doi:10.1109/ LCOMM. 2002.806451

[13] J. K. Cavers and M. W. Liao, "Adaptive Compensation for Imbalance and Offset Losses in Direct Conversion Transceivers," IEEE Transaction on Vehicular Technol- ogy, Vol. 42, No. 4, 1993, pp. 581-588. doi:10.1109 $/ 25.260752$

[14] S. Haykin, "Unsupervised Adaptive Filtering, Vol. I: Blind Source Separation," John Wiley \& Sons, New York, 2000.

[15] S. Haykin, "Unsupervised Adaptive Filtering, Vol. II: Blind Deconvolution," JohnWiley \& Sons, New York, 2000.

[16] A. Hyv"arinen, J. Karhunen and E. Oja, "Independent Component Analysis," JohnWiley \& Sons, New York, 2001. doi:10.1002/0471221317

[17] J. Eriksson and V. Koivunen, "Identifiability, Separability, and Uniqueness of Linear ICAmodels," IEEE Signal Processing Letters, Vol. 11, No. 7, 2004, pp. 601-604. doi:10.1109/LSP.2004.830118

[18] J.-F. Cardoso, "Blind Signal Separation: Statistical Principles," Proceeding of the IEEE, Vol. 86, No. 10, 1998, pp. 2009-2025. doi:10.1109/5.720250

[19] J.-F. Cardoso and B. H. Laheld, "Equivariant Adaptive Source Separation," IEEE Transaction Signal Processing, Vol. 44, No. 12, 1996, pp. 3017-3030. doi:10.1109/78. 553476

[20] S. Amari, S. C. Douglas, A. Cichocki and H. H. Yang, "Multichannel Blind Deconvolution and Equalization using the Natural Gradient," 1st IEEE Proceeding on Workshop on Signal Processing Advances in Wireless Communications, Paris, April 1997, pp. 101-104. 